\title{
Tsallis scaling in the long-range Ising chain with competitive interactions
}

\author{
E. M. Carvalho Neto and R. F. S. Andrade \\ Instituto de Física, Universidade Federal da Bahia, 40210-340 Salvador, Brazil
}

(Received on 19 January, 2009)

\begin{abstract}
A numerically efficient transfer matrix approach is used to investigate the validity of the Tsallis scaling hypothesis in the long-range Ising spin chain with competitive interactions. In this model, the interaction between two spins $i$ and $j$ placed $r$ lattice steps apart is $J_{i, j}=(-1)^{\zeta(i, j)} J_{0} / r^{\alpha}$, where $\zeta(i, j)$ is either 0 or 1 . This procedure has succeeded to show the validity of the scaling hypothesis for the well investigated ferromagnetic version of the model, i.e., $\zeta(i, j)=0, \forall i, j, \forall \alpha \geq 0$. Results are reported for some models of a set, which is defined by requiring $\zeta(i, j)$ to be a periodic sequence of $0^{\prime} s$ and $1^{\prime}$ s. As expected from symmetry arguments, we find that the hypothesis is not valid when $\zeta(i, j)=1, \forall i, j$ and $\alpha<1$. However, it is verified, with high degree of numerical accuracy, when $\alpha<1$, for sequences in which the occurrence of $\zeta(i, j)=0$ is more frequent than that of $\zeta(i, j)=1$.
\end{abstract}

Keywords: Competitive interactions, Long-range Ising chain

\section{INTRODUCTION}

The properties of the ferromagnetic long-range Ising chain have been investigated by a large number of authors in the past five decades [1-10]. However, the anti-ferromagnetic and other more general versions of the model, where both ferro and anti-ferromagnetic interactions are present, have been almost neglected by the statistical physics community. A possible explanation for this is the fact that, in opposition to the ferromagnetic case, the cumulative effect of long-range anti-ferromagnetic interaction is not of producing cooperative effect responsible for spin ordering even in the linear chain. Therefore, such system is not likely to produce any critical behavior. Another possible reason to the lack of interest in the model is the known mathematical and computational difficulties that one must overcome to obtain results already for the simple ferromagnetic model.

A few years ago, one of us has developed an alternative transfer matrix (TM) approach to investigate the thermodynamical properties of the ferromagnetic Ising chain, which is suitable to produce very accurate numerical results. Besides that, it can be numerically implemented in relatively small computational facilities [11]. The procedure, which amounts to working with small TM's, is optimized with respect to storing the energy values for all distinct spin configurations and to avoiding the numerical evaluation of the TM largest eigenvalue. Within this framework, interactions among spins placed up to $g=25$ lattice sites apart could be taken into account also in the evaluation of the critical properties [12]. When discussing the results for the thermodynamic function, special attention was devoted to verifying the validity of the Tsallis scaling hypothesis [13, 14], which had not been addressed in the investigations prior to 1995 .

It is worthy stressing that, besides the direct evaluation of the thermodynamical properties of long range magnetic models by ensemble averages, an alternative approach to this classical problem has been followed by several authors for about a decade. It refers to evaluating time average of the corresponding quantities, by numerically integrating the equations of motion that follow from the system's Hamiltonian. The time integration approach, which seems more adequate for the models with continuous valued variables, as the $X Y$ spin model [15], has been mainly motivated by the problem of identifying whether long range models may be described by the non extensive statistical mechanics [16].

This work has two main purposes. The first one, which has a more technical aspect, is to adapt the previously developed TM approach and obtain the thermodynamical properties of Ising chains with the presence of both ferro and anti-magnetic interactions. The second one is to verify the validity of Tsallis conjecture [14] for a broad class of models, which can be written in terms of the following spin Hamiltonian:

$$
\mathcal{H}=-\sum_{i=-\infty}^{\infty} \sum_{r=i+1}^{\infty} J_{r} \sigma_{i} \sigma_{i+r}-H \sum_{i=-\infty}^{\infty} \sigma_{i}
$$

In Eq. (1), $J_{r}=J_{i, j}=(-1)^{\zeta(i, j)} J_{0} / r^{\alpha}, j=i+r$, and $\zeta(i, j)=0$ or 1 controls the ferro- or anti-ferromagnetic character of the interaction between spins $i$ and $j$. Note that, since $J_{i, j}$ depends only on the distance between spins $i$ and $j$, the models that can be described by Eq. (1) are homogeneous in space. Even though, many distinct situations can be described by the function $\zeta(i, j)$. In this work, we restrict our investigation to a restricted class of $\zeta(i, j)$, namely, those periodic sequences of $0^{\prime} \mathrm{s}$ and $1^{\prime} \mathrm{s}$ that can be expressed in terms of $F(k, \ell)$ and $A(k, \ell)$ as follows:

$$
\begin{aligned}
& F(k, \ell): \zeta(i, i+r)=0, \text { for } 1 \leq \underline{r} \leq m \\
& \zeta(i, i+r)=1 \text {, for } m+1 \leq \underline{r} \leq n \\
& A(k, \ell): \zeta(i, i+r)=1, \text { for } 1 \leq \underline{r} \leq m \\
& \zeta(i, i+r)=0, \text { for } \quad m+1 \leq \underline{r} \leq n \\
& \underline{r}=r \bmod (m+n+1) .
\end{aligned}
$$

Due to the dependence of $J_{r}$ on $r$, the frequency of values of 0 's or 1's and the relative strength of the corresponding bonds impose different dominant ferro or anti-ferromagnetic character to the $F(k, \ell)$ and $A(k, \ell)$ defined models. The pure ferro- or anti-ferromagnetic models, corresponding to $\zeta_{i, i+r}=$ 0 or $1, \forall r$ situations, become degenerated in the $F(k, \ell)$ and $A(k, \ell)$ definitions. For the sake of simplicity, these limit cases will be denoted by $F(1,0)$ and $A(1,0)$.

The rest of this work is organized as follows: in the Sec. II we present the brief discussion of the expressions that define the Tsallis scaling while, in Sec. III, we summarize the TM method used to evaluate the partition function and the 
free energy. Results are presented and discussed in Sec. IV. There we indicate, based on the behavior of the free energy, the parameter range where we observe the validity of Tsallis scaling. Finally, conclusions and perspectives of future works are discussed in Sec. V.

\section{THE TSALLIS SCALING CONJECTURE}

The Tsallis scaling conjecture (TSC) [13, 14, 17, 18] addresses, in an adequate way, the problem of defining any intensive energy-like thermodynamical property for $d$-dimensional systems, where the energy interaction decreases with the distance $r$ as $r^{-\alpha}$. Due to the presence of long-range interactions, measured by the ratio $\alpha / d$, the total energy $U$ may increase faster than the number $N$ of constituents, i.e., the usual ratio $U / N$ diverges in the $U, N \rightarrow \infty$ limit. TSC generalizes, for instance, the mean field results for the free Gibbs energy of a homogeneous ferromagnetic system with constant interaction among any pair of spins, where the ratio between the intensive function $f(T, H, N)$ and the $\operatorname{logarithm}$ of the partition function, $\log Z(T, H, N)$, is $1 / N^{2}$.

The TSC for Gibbs free energy is synthesized by the following expression, where tildes indicate the re-scaled intensive quantities:

$$
\tilde{f}(\tilde{T}=T / \tilde{N}, \tilde{H}=H / \tilde{N})=f(T, H, N) / \tilde{N},
$$

where $H$ denotes the magnetic field and the scaling variable $\tilde{N}$ is defined as

$$
\tilde{N}=\frac{N^{1-\alpha / d}-\alpha / d}{1-\alpha / d} .
$$

In the $N \rightarrow \infty$ limit, $\tilde{N}$ may be simplified by the following expressions, which depend on the ratio between the system dimensionality $d$ and the exponent $\alpha$ :

$$
\tilde{N}=\left\{\begin{array}{ll}
\frac{\alpha / d}{\alpha / d-1} & \text { if } \quad \alpha / d>1 \\
\ln N & \text { if } \quad \alpha / d=1 \\
\frac{1}{1-\alpha / d} N^{1-\alpha / d} & \text { if } \quad 0 \leq \alpha / d<1
\end{array} .\right.
$$

The different dependence of $\tilde{N}$ on $\alpha$ clearly distinguishes the extensive region $(\alpha / d>1)$, where $\tilde{N}$ becomes independent of $N$ in the limit $N \rightarrow \infty$, from the non-extensive region, where $\tilde{N}$ explicitly depends on $N$ in the same limit.

TSC states further that other $N$-dependent intensive thermodynamical functions, like the entropy $s(T, H, N)$, specific heat $c(T, H, N)$ and magnetization $m(T, H, N)$, have their $N$ independent counterparts $\tilde{s}, \tilde{c}$, and $\tilde{m}$, defined by

$$
\begin{aligned}
\tilde{s}(\tilde{T}, \tilde{H}) & =s(T, H, N), \\
\tilde{c}(\tilde{T}, \tilde{H}) & =c(T, H, N), \\
\tilde{m}(\tilde{T}, \tilde{H}) & =m(T, H, N) .
\end{aligned}
$$

The TSC applies only to situations where the "effective" range of interaction decreases as a power law. For instance, screening effects reduces the effective range of the interaction, of systems of positive and negative charges interacting via the long-range Coulomb interaction. In such situations, intensive energies are obtained just by dividing the total energy by $N$, not by $N^{2}$. A similar effect is observed in spin systems where all long-range couplings are anti-ferromagnetic. Here, the screening effect is replaced by bond frustration that results from the competing spin interactions. Therefore, intensive energies are obtained by dividing the total energy of lowest-energy spin configurations by $N$.

Intensive energies of mixed systems, in which long interactions of alternating signs coexist, may require the introduction of TS. This will be explored in Sec. IV, where we concentrate on a few situations described by $F(k, \ell), k \geq \ell$, sequences.

Before closing this section, we would like to state that TSC gave rise to a series of investigations to test its validity. Several long-range interaction models have been studied by different methods. They include spin models in connection with mean-field, renormalization group and Monte Carlo simulation [19-23] .

\section{TRANSFER MATRIX FORMULATION}

Since one must resort to numerical evaluation of the thermodynamical properties, we are forced to describe the infinite system described by Eq.(1) with help of the finite size approximations, which may depend both on the total number $N$ of spins in the chain, and on the maximal range $g$ of interaction couplings. Thus, we consider a finite chain with $N=g+c+1 \geq g+1$ spins, and $g$ distinct coupling constants $J_{r}, r=1,2, \ldots g$. Fixing the value of $g$, we notice that the inclusion of $c$ new spins in the chain amounts to add $g \times c$ new interactions. In Figure 1 we show, for the purpose of illustration, the interactions for the first 3 values of $c$ for fixed $g=3$.

Although the method we use herein has been discussed in detail elsewhere $[11,12]$, let us briefly comment on the most relevant steps that allow the evaluation of the partition function. Thus, let us define a $2 \times 2 \mathrm{TM} M_{g, c}$ by

$$
M_{g, c}=\left(\prod_{k=1}^{g} P_{k}\right)\left(Q_{g} \cdot P_{g}\right)^{c} \cdot L_{g} \equiv R_{g, c} L_{g} .
$$

$L_{g}$ denotes a $2^{g+1} \times 2$ matrix with elements $\left(L_{g}\right)_{i, j}=1(0)$, when $i+j$ is even (odd). The matrix elements of the $2^{g+1} \times 2^{g}$ matrix $Q_{g}$ are defined by

$$
\left(Q_{g}\right)_{i, j}=\left\{\begin{array}{ll}
1 & \text { for } i=j \text { or } i=j+2^{g} \\
0 & \text { otherwise }
\end{array} .\right.
$$

On the other hand, the matrix elements of the auxiliary matrices $P_{k}$ are recursively defined by:

$$
\left(P_{k}\right)_{i, j}= \begin{cases}\left(P_{k-1}\right)_{i, j} a_{k}^{(-1)^{j-1}} & \text { for } i \leq 2^{k-1} \\ \left(P_{k}\right)_{2^{k}-i+1,2^{k+1}-j+1} & \text { and } j \leq 2^{k} \\ & \text { for } 2^{k-1} \leq i \leq 2^{k} \\ 0 & \text { and } 2^{k} \leq j \leq 2^{k+1} \\ \text { otherwise }\end{cases}
$$

where $a_{k}=\exp \left(J_{k} / T\right)$ are the problem Boltzmann weights.

These expressions lead to the partition function

$$
Z_{g, c}=2 \lambda_{g, c}^{+}=2\left(\left(M_{g, c}\right)_{1,1}+\left(M_{g, c}\right)_{1,2}\right)=\sum_{i, j}\left(R_{g, c}\right)_{i, j}
$$




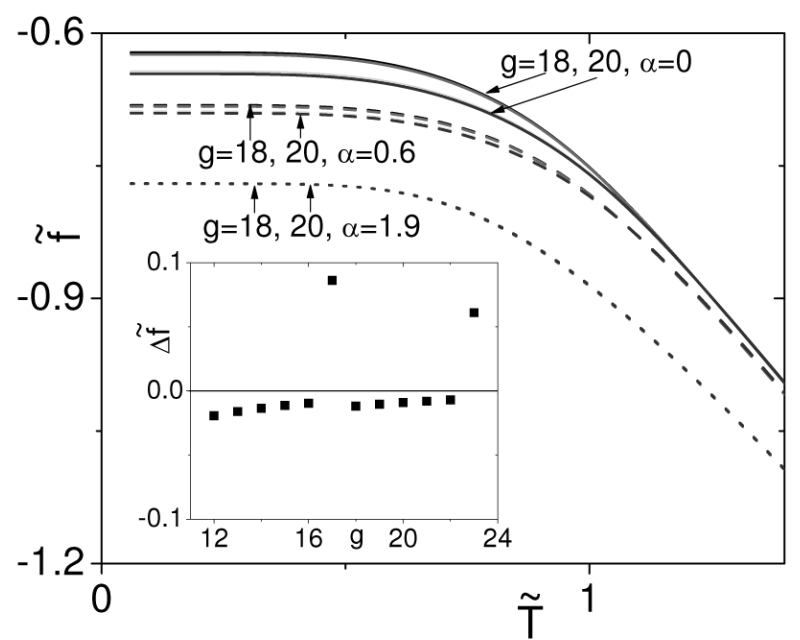

FIG. 1: Model $F(5,1)$ : TS free energy $\tilde{f}$ for $\alpha=0.0$ (solid), 0.6 (dashes), and 1.9 (dots), when $c=90,95$, and $g=18,20$. Good convergence of $\tilde{f}$ with respect to $c$ is observed for all values of $\alpha$. Convergence with respect to the values of $g$ weakens as $\alpha$ decreases. In the inset, differences $\Delta_{g, \delta} \tilde{f}$ when $\alpha=0.0, c=95, \delta=1$, as function of $g \in[12,23]$, for $\tilde{T}=0.02$. Periodic oscillations are related to appearance of a $J<0$ at every sixth value of $g$, when the magnitude of $\Delta_{g, 1} \tilde{f}$ increases and changes sign. After such jumps, the decreasing tendency is resumed, indicating that $\tilde{f}$ converges for all values of $\alpha$.

and to the free energy per spin, $f_{g, c}=-T \ln \left(Z_{g, c}\right) / N$.

From Eq.(10) it is possible to obtain expressions for the derivatives of $f$ with respect to $T$ and $H$ in terms of proper sums of Boltzmann weights. This way, thermodynamic functions like $s, c$ and $m$ can be evaluated with high degree of precision, avoiding numerical differentiation procedures.

\section{TS AND FREE ENERGY}

We discuss results obtained by the method described in the previous sections for chains with coupling constants introduced according to the following sequences: $F(2,1), F(4,1)$, $F(5,1), A(1,0)$, and $A(1,1)$.

Since the focus of this work is the scaling properties of the free energy, our discussion is based on numerical evidences that support the validity of TS for several of the quoted sequences and values of $\alpha$. However, for some other specific sequences, the results indicate that the free energy is extensive, even for values of $\alpha$ where the ferromagnetic model is non-extensive. This evidences a break of validity of TS, since Eq. 3 no longer holds.

Let us start by considering the $F(k, 1)$ sequences. We think it is more suitable to start the discussion with the behavior of the system for large values of $m$, as the pure ferromagnetic $F(1,0)$ chain can be reproduced when the limit $k \rightarrow \infty$ is considered. We have found that the values $k \leq 5$ are sufficient large to provide a clear picture of the validity of TSC. Our results have been summarized in Figs. 2-5, which show the behavior of $\tilde{f}$ as function of $\tilde{T}$ for decreasing values of $k$.
In order to test the validity of TS, it is important to call the attention to the different roles played by $g$ and $N$ in our approach. As explained in Sec. 2, it allows the possibility of working with a number $N$ of constituents larger than the maximal distance $g$ between interacting spins. This contrasts with the TS definition based on Eq. (4), which makes no distinction between $N$ and $g$. As discussed previously [11], it turns out that $g$, instead of $N$, is the relevant parameter to be substituted in all Tsallis scaled quantities. This is related to the fact that TS accounts for the range $g$ of interactions, while the number of constituents $N$ is accounted for by dividing the total energy by $N$. This has been made clear in the results for the $F(1,0)$ Ising chain, where one easily notes that, for small values of $g$, the curves for $f$ at different values of $c$ are Tsallis scaled as distinct systems.

Fig. 1 illustrates, for the $F(5,1)$ sequence, the dependence of $\tilde{f}$ on $g=18$ and 20 , for fixed values of $c=90,95$, when $\alpha=0.0,0.6$, and 1.9. The collapse for the different sets of 4 curves have quite good quality with respect to the dependence on $c$, but the picture changes when we consider the difference with respect to $g$. First note that, the 4 curves for $\tilde{f}$ in the extensive region $(\alpha=1.9)$ are almost indistinguishable. Regarding the dependence on $g$, we recall that for large values of $g$, TS amounts only to scaling both axes by an $g$ independent factor. Thus, the corresponding plots actually show the dependence of both $f$ and $\tilde{f}$ on $g$ and, as a consequence, the validity of TS in this particular range is an expected result. For the values of $\alpha$ in the non-extensive region, we notice that the convergence of $\tilde{f}$ for $\alpha=0.6$, measured by the difference between the curves for $g=18$ and 20 is not so fast. This effect is related to the stronger dependence of $f$ and $\tilde{N}$ on $g$ for smaller values of $\alpha$, and has also been found for the $F(1,0)$ chain. The convergence is actually much more complex, as can be quantitatively estimated by evaluating the differences $\Delta_{g, \delta} \tilde{f}=\tilde{f}_{g+\delta, c}(\tilde{T})-\tilde{f}_{g, c}(\tilde{T})$. For the sake of a better visualization, we draw in the inset values of $\Delta_{g, \delta}$ for $\delta=1$ as function of $g \in[12,23]$, for a fixed value of $\tilde{T}=0.02$. We clearly see an oscillating behavior of period 6 , which is related to the introduction of bonds with $J<0$. Whenever this occurs, the total energy of the system is reduced in comparison to the previous value of $g$, causing a jump in the value of $\Delta_{g, \delta}$. After such jumps, the values of $\Delta_{g, \delta}$ become negative while its absolute value has again decreasing tendency. It is also possible to notice that the height of such jumps also decreases as $g$ increases.

Such effects are enhanced for $\alpha=0.0$, in the sense that both the absolute values of $\Delta_{g, \delta}$ in the decreasing region, as well as the height of the jumps, are larger. Nevertheless, they keep showing the monotonic behavior. Such results can be compared with those obtained for the $F(1,0)$ chain where TS is valid. This is a clear indication that, despite slower convergence, TS holds for the wide range of values of $\alpha$.

Increasing the relative presence of AF bonds in the chain affects the behavior of $\Delta_{g, \delta} \tilde{f}$ in a variety of ways, which clearly depend on the value of $\alpha$ and $k$. The changes affect both the convergence properties as well as the period of the oscillations. This is illustrated in Figs. 2 and 3 for models described, respectively, by the $F(4,1)$ and $F(2,1)$ sequences. We consider $g=17,18,19,20,21$, the same values of $c$ as in Fig. 1 , while $\alpha=0.0$ and 0.6. Since the behavior of $f$ and $\tilde{f}$ in the extensive region is much like that one for the $F(5,1)$ 


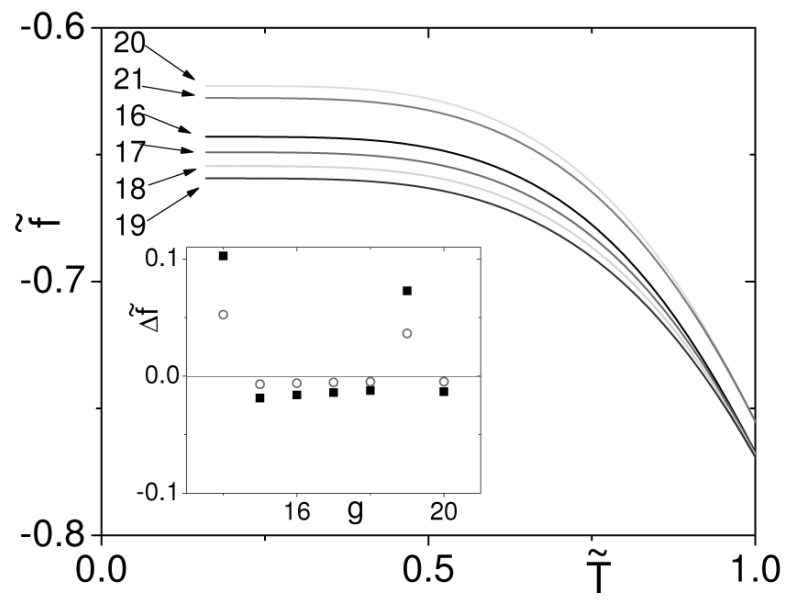

FIG. 2: Model $F(4,1)$ : TS free energy $\tilde{f}$ for $\alpha=0.6, c=95$, while $g=16,17,18,19,20$ and 21 . For the whole range of values of $\tilde{T}$, the curves clearly change their tendency of getting together when a $J<0$ is introduced in the chain. The period of oscillations is reduced to 5. In the inset, differences $\Delta_{g, \delta} \tilde{f}$ when $c=95, \delta=1$ and $g=$ $16,17,18,19$ and 20 . The behavior of $\Delta_{g, \delta} \tilde{f}$ suggests TS is valid for both values of $\alpha=0.0$ (back squares) and 0.6 (red open circles).

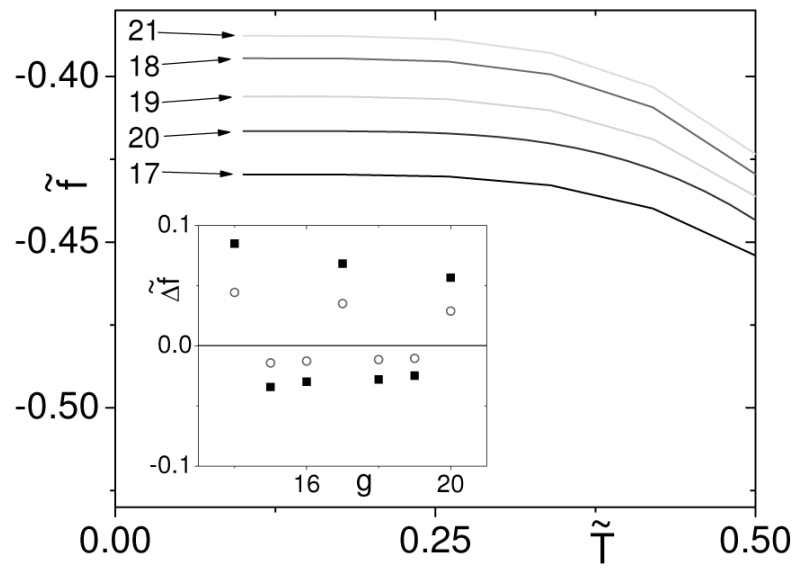

FIG. 3: Model $F(2,1)$ : TS free energy $\tilde{f}$ for the same values of $g$ as in Fig. 2, with exception of $g=16$. The magnitudes of $\Delta_{g, \delta} \tilde{f}$ (inset), for the same values of $\alpha$ as in Fig. 2 are considerably larger. The overall decreasing tendency suggests validity of TS for both values of $\alpha$. The oscillation period 3 fits well into the overall frame discussed before.

model, the discussion is restricted to the analysis of convergence in the non-extensive range of $\alpha$.

The features in Figs. 2 and 3 confirm the overall tracts described before. By comparing the explicit temperature dependence of the curves for $\alpha=0.6$ we note a decrease in the absolute value of $\tilde{f}$ when a negative coupling constant is introduced at $g=15$ and 20 for $F(4,1)$, and at $g=15,18$ and 21 for $F(2,1)$. The tendency of an oscillatory dependence of $\Delta_{g, \delta} \tilde{f}$ with respect to $g$ is observed again, but the period

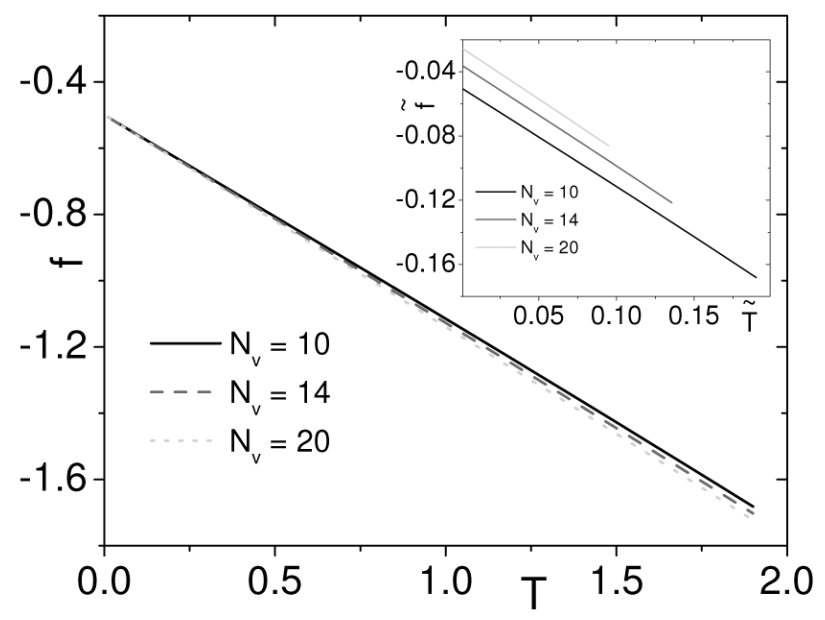

FIG. 4: Model $A(1,0)$ : Usual free energy $f=F / N$ as function of $T$ for $\alpha=0, c=0, g=10,14,20$. The curve collapse indicates that $f$ is intensive. The inset shows that TSC is not valid, as the corresponding $\tilde{f}$ curves do not collapse.

is reduced, respectively, to 5 and 3 . After each jump, $\Delta_{g, \delta} \tilde{f}$ decreases monotonically with $g$, much as observed in Fig.1.

Now let us consider the two $A$ sequences. Due to high configurational symmetry, the models defined by the selected sequences are amenable to exact results in some parameter and temperature ranges. If we choose an arbitrary spin in the pure $\mathrm{AF}(A(1,0))$ model, its minimum energy state occurs when it is anti-parallel to all other spins. As this happens to any spin, we immediately see that a large number of frustrated bonds necessarily occurs in the large degeneracy ground state. When $\alpha=0$, it is possible to exactly compute the ground state energy $E_{0}=-J_{0} N / 2$ and degeneracy $N ! /[(N / 2) !(N / 2) !]$. This shows that the free energy is extensive, and that entropy per spin $s=k_{B} \ln 2$ is temperature independent. As advanced in Sec. 2, this shows that TS does not apply. We observe that no magnetization or sub-lattice staggered magnetization are observed, even at $T=0$. In the other $\alpha \rightarrow \infty$ limit situation, the ground state of the system is characterized by a simple minimum energy configuration consisting of anti-parallel neighboring spins. In such situation, TS is trivially verified as $\tilde{N}=1$.

Such features were reproduced by the numerical evaluation of the partition function. In Fig.4, the quite good collapse of curves for $g=10,14$ and 20 make it clear that the free energy $f=F / N$ is an intensive quantity. In the inset, the $\tilde{f} \times$ $\tilde{T}$ curves for the same values of $g$ confirm that, as expected, TSC is not valid for the current model.

The situation changes continuously for intermediate values of $\alpha$ in the interval $(0,1)$. Although we cannot compute exactly the value of the ground state energy, the numerical evaluation of $Z$ confirms that $f$ is intensive, as shown in Fig.5 for $\alpha=0.2$ and the same values of $g$ as in Fig.4. The curves in Figs. 4 and 5 were obtained by considering $c=0$. Indeed, the exact results discussed above for $\alpha=0$ require that all spins interact among themselves. If we take $c>0$, this requirement is no longer met and, as a consequence, the collapse of the 


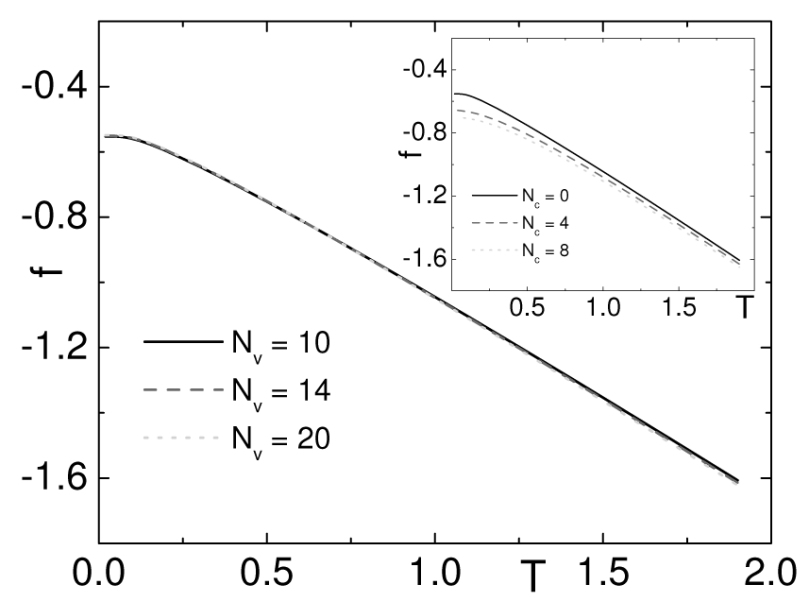

FIG. 5: Model $A(1,0): f$ as function of $T$ for $\alpha=0.2, c=0, g=$ $10,14,20$. The collapse confirms that the free energy is extensive in the whole range of $\alpha$ values. The inset shows the dependence of $f$ on values of $c>0$, when fixing the value $g=10$.

different $f$ curves, especially in the low temperature region, is not observed. This is exemplarily shown in the inset of Fig.5.

The next sequence, $A(1,1)$, leads to a situation that can be understood on the basis of the traditional ferromagnetic chain. Indeed, the sequence indicates that the model is composed of constant couplings of alternating signs, starting with a $J_{r=1}<0$ : spins in neighboring sites have a tendency to align anti-parallel to each other. As the second-neighbor coupling $J_{2}>0$, this results no competition between the two bonds. The same is valid for all other values of $r$ and, as a result, the system is decomposed into two sub-lattices, which are distinguished only by the fact that the spins align in opposite direction. Therefore, the total energy increases faster than the number of constituents, and TS is verified in a similar way as observed for the quoted $F(1,0)$ chain.

Finally, let us comment on the scaling properties of the model described by the $F(1,1)$ sequence. If we consider $\alpha=0$, it is clear that frustration occurs for a very large number of bonds. The situation is much similar to the one we find for the already discussed $A(1,0)$ sequence. There, all pairs of spins $\sigma_{i} \sigma_{i+r}$ separated by even (odd) $r$ would like to point in the same (opposite) directions. In the $F(1,1)$ case, the situation is the opposite, i.e., all pairs of spins separated by even (odd) $r$ would like to point in the opposite (same) directions. This indicates that energy is extensive, entropy is constant, and TS is not valid.

\section{CONCLUSIONS}

In this work we presented some preliminary results on the behavior of the Ising chain with the presence of long-range interactions of competing character. Our main focus was to establish for which parameter interval the free energy of the system continues to follow the scaling properties proposed by Tsallis.

Our results were obtained within a previously introduced framework for the analysis of the simplest ferromagnetic version of the general problem. The procedure, which can be formulated with the help of transfer matrices, amounts to the precise numerical evaluation of the partition function $Z(T, N=g+c+1)$, where the range of interaction and number of spins in the system have been as large as 24 and 200, respectively.

We selected five different systems, which distinguish themselves by the order in which interactions with coupling couplings $J>0$ and $J<0$ are introduced. We also discussed some limiting situations which, due to high symmetry, are amenable for analytical results.

In the extensive region $\alpha>1$, the value of $\tilde{N}$ approaches a constant value when $N \rightarrow \infty$, what has less impact in the search of an intensive free energy. Because of that, we concentrate our discussion of the validity of TS in the nonextensive region $0 \leq \alpha<1$.

As a general result, we have shown that continuously increasing the number of AF interactions on a basic model where, initially, all $J>0$ contribute to decrease the speed at which TS is attained. We have uncovered the emergence of periodic oscillations in the convergence process of $\tilde{f}$. Despite the reduction in the convergence, our results show that, as long as the number of $\mathrm{F}$ bonds exceeds that of AF counterparts, the validity of TSC is observed.

On the other hand, systems where all $J<0$ do not obey TS when $0 \leq \alpha<1$. Such result was obtained from exact counting and confirmed by the numerical evaluation of the partition function.

The situation when the number of $\mathrm{F}$ and $\mathrm{AF}$ bonds is equal has also been addressed. We have indicated that the model described by the $A(1,1)$ sequence is analogous to the pure ferromagnetic chain $F(1,0)$. Thus, the total free energy is non-extensive and TS is valid. Much in a symmetric way, the behavior of the $F(1,1)$ model is analogous to the pure ferromagnetic chain $A(1,0)$ : the free energy is extensive and TS is not valid for $\alpha=0$.

\section{Acknowledgements}

This work was partially supported by the Brazilian agencies CNPq and CAPES. The authors acknowledge useful discussions with S.T.R. de Pinho. 
[3] J. L. Cardy, J. Phys. A 14, 1407 (1981).

[4] M. Aizenman, J. T. Chayes, L. Chayes, and C. M. Newman, J. Stat. Phys.50, 1 (1988); M. Aizenman and R. Fernández, Lett. Math. Phys. 16, 39 (1988).

[5] J. F. Nagle and J. C. Bonner, J.Phys. C 3, 352 (1970).

[6] M. E. Fisher, S. K. Ma and B. G. Nickel, Phys. Rev. Lett. 29, 917 (1972).

[7] J. L. Monroe, R. Lucente, and J. P. Hourlland, J. Phys. A 23, 2555 (1990).

[8] Z. Glumac and K. Uzelac, J. Phys. A 22, 4439 (1989).

[9] Z. Glumac and K. Uzelac, J. Phys. A 26, 5267 (1993).

[10] S. A. Cannas and A. C. N. de Magalhães, J. Phys. A 30, 3345 (1997).

[11] R. F. S. Andrade and S. T. R. Pinho, Phys. Rev. E 71, 026126 (2005).

[12] R. F. S. Andrade and S. T. R. Pinho, Eur. J. Phys. B 50, 33
(2006).

[13] P. Jund, S. G. Kim, and C. Tsallis, Phys. Rev. B 52, 50 (1995).

[14] C Tsallis, Fractals 3, 541 (1995).

[15] C. Anteneodo and C. Tsallis, Phys. Rev. Lett. 80, 5313 (1998).

[16] C. Tsallis, J. Stat. Phys. 52, 479 (1988).

[17] C. Tsallis, Braz. J. Phys. 29, 1 (1999).

[18] S. Abe and A.K. Rajagopal, Phys. Lett. A 337, 292 (2005).

[19] F. Tamarit and C. Anteneodo, Phys. Rev. Lett. 84, 208 (2000).

[20] H. H. A. Rego, L. S. Lucena, L. R. da Silva, and C. Tsallis, Physica A 266, 42 (1999).

[21] S. A. Cannas, Phys. Rev. B 52, 3034 (1995).

[22] S. A. Cannas and F. A. Tamarit, Phys. Rev. B 54, R12661 (1996).

[23] S. A. Cannas, A. C. N. de Magalhães, and F. A. Tamarit, Phys. Rev. B 61, 11521 (2000). 